\title{
SIMILARITY THEORY OF SOLAR WATER HEATER WITH NATURAL CIRCULATION
}

\author{
B. J. HUANG \\ Department of Mechanical Engineering, National Taiwan University, Taipei, Taiwan, Republic of China
}

(Received 27 June 1979; revision accepted 11 December 1979)

\begin{abstract}
The similarity theory of solar thermosyphon collector is developed in the present paper. Ten dimensionless groups or system characteristic parameters which uniquely determine the performance of the collector are derived. The solution shows that the mean efficiency generally increases with increasing incident solar radiation and relative height of the tank. For the frictional parameters $N_{e}$ and $N_{f}$ higher than $10^{5}$, the efficiency appears to be independent of the incident radiation and the relative height of the tank. Therefore, for parallel plate absorber, the tank may be designed to sit on the floor without sacrificing the efficiency since the values of $N_{e}$ and $N_{f}$ are usually larger than $10^{5}$ in most designs.
\end{abstract}

\section{INTRODUCTION}

A solar hot water heater with natural circulation is now one of the most economic devices for collecting solar energy for domestic or industrial uses. Many theoretical and experimental work have been carried out during the past decades to study the thermal performance of the collector. A common conclusion deduced from a great deal of experiments performed by many investigators indicate that for better performance the relative height of the storage tank above the absorber generally must be higher than thirty centimeters for the ordinary collector sizes being tested [1]. For different collector designs or largescale collectors which might be used in large-scale applications, it is believed that this design criteria would change. However, little has been known about this.

A mathematical model which introduces a mean system temperature and assumes linear temperature distributions in absorber plate and storage tank was first proposed by Close [2] to predict the collector performance. Further improvements are made by Gupta and Garg [3] later and more recently by Ong [4,5]. Gupta and Garg [3] improved Close's analysis by incorporating a plate efficiency factor to account for the thermal efficiency of the plate and considering the variations of ambient temperature and radiation intensity. Ong [4] further carefully considered the plate efficiency factor and also assumed linear temperature distributions in absorber plate and storage tank. A finite difference method was then employed to solve the governing equations. Another modification was again made by Ong [5]. Instead of assuming linear temperature distribution, Ong further considered the entire system to be broken up into a finite number of sections each individual section having a uniform mean temperature, then solved the finite difference equations. The solutions were found in very good agreement with experimental results. The theoretical analyses mentioned above however can only predict the performance for a specified collector with known dimensions. No general design rules can be drawn from the above analyses to tell what is the effect of the relative height of the storage tank above the absorber plate on the performance for different sizes of collector.
Only little evidence was noticed by Ong [5] in his experiment which indicates that the mean collector efficiency increases with increasing relative height of tank as well as solar radiation intensity.

Grossman et al. [6] attempted to normalize the governing equations of the collector to obtain similarity solutions for a parallel-plate type absorber itself. For simplicity, they assumed that the performance is quasisteady with a known function of net heat flux distribution reaching the fluid through the upper plate and ignored heat transfer and frictional loss in the connecting pipes and the storage tank. Therefore, the analytical results only give a very rough picture of the collector performance. The effect of the relative height of the storage tank above the absorber plate as well as the frictional loss on the system performance was still not quite clear.

The present work attempts to develop a more general similarity theory by a model which divides the entire collector into three components: absorber plate, storage tank and connecting pipes. Two energy equations for absorber and storage tank and a momentum equation for the entire flow system are derived. The governing equations are then normalized to come up with ten dimensionless groups which characterize the performance of thermosyphon collector. The similarity solutions thus give a very clear picture on the collector performance for any size of collector and result in some important design rules.

\section{MATHEMATICAL MODEL}

The thermosyphon solar collector studied in the present paper basically consists of three major components: namely, absorber plate, storage tank and connecting pipes (see Fig. 1). The incident solar energy radiation is absorbed by the blackened surface of the absorber plate and transmitted through the plate material to the fluid. The temperature rise of the fluid inside the absorber then causes to a density decrease and induces a driving force between the absorber side (hot side) and the storage tank side (cold side) due to the density difference. By this driving force, called thermosyphon head, the fluid circulates upward from the absorber 

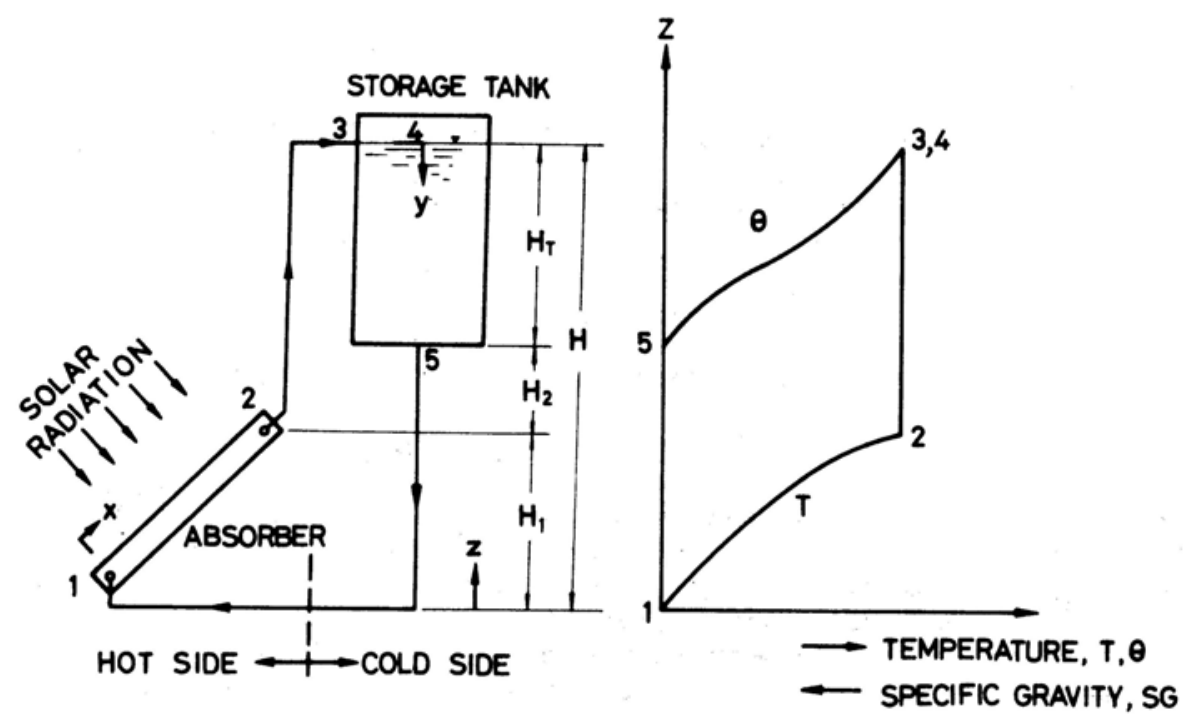

Fig. 1. Schematic diagram of thermosyphon solar collector and temperature distribution curve.

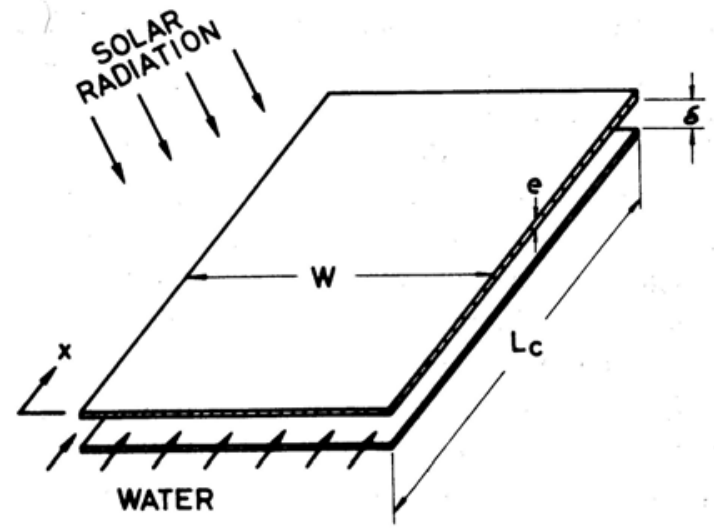

Fig. 2. Schematic diagram of parallel-plate absorber.

through the storage tank and returns to the absorber. For simplicity, we assume that no water is drawn off from the storage tank during daytime although hot water drawn-off would significantly affect the performance of the collector. Moreover, the absorber design is assumed to be of parallel plate type as shown in Fig. 2 in the present analysis.

To facilitate the analysis, some assumptions are made:

(1) The velocity and temperature distributions of the fluid in the storage tank are approximately one-dimensional $[7,8]$.

(2) The velocity and temperature distributions inside the absorber plate are one-dimensional.

(3) The transient behavior of the heat transfer inside the connecting pipes is ignored.

(4) The storage tank and the connecting pipes are well-insulated therefore the heat loss from them are negligible.

(5) The effect of the heat capacity due to the constructing material of the whole system can be ignored.

(6) The flows in the tank, the connecting pipes and the absorber are laminar since the Reynolds number is usually smaller than $\mathbf{3 0 0 0}$ in most applications.

(7) The heat conduction along the flow direction in the absorber is negligible since the Peclet number is usually large.
(8) No water drawn-off during daytime is allowed.

(9) The incoming solar energy received and actually converted into heat energy at the black surface of the absorber can be approximately represented by a sine function, i.e. $S=G_{m} \sin (\pi t / \omega)$ where $\omega$ is the total sunshine duration, $G_{m}$ is the radiation intensity at solar noon.

\section{Energy balance}

For parallel-plate absorber considered in the present paper (Fig. 2), taking an energy balance on an element of fluid inside the absorber yields the energy equation:

$$
\frac{\partial T}{\partial t}+\left(\frac{q L_{c}}{\delta}\right) \frac{\partial T}{\partial x}=\frac{F^{\prime}}{\rho C_{p} \delta}\left[G_{m} \sin (\pi t / \omega)-U_{L}\left(T-T_{a}\right)\right]
$$

where

$$
\begin{aligned}
F^{\prime} & \equiv \frac{h_{i}}{U_{L}\left(1+B_{i}\right)+h_{i}}, \\
B_{i} & \equiv h_{i} e / k_{c}, \\
q & \equiv v_{c} \delta / L_{c} .
\end{aligned}
$$

Here, $F^{\prime}$ is the absorber efficiency factor which depends on the collector design, $v_{c}$ is the mean velocity inside the absorber, $B_{i}$ is the Biot number which represents the ratio of heat convection to the heat conduction between the absorber plate and the fluid, and $q$ represents the volumetric flowrate per unit area of collector. $U_{L}$ is the overall heat loss coefficient from the black surface of the absorber to the ambient, $T_{a}$ is the ambient temperature which is assumed constant in the present analysis, $T$ is the local fluid bulk temperature. The above equation can be normalized to the form:

$$
N_{c} \frac{\partial T^{*}}{\partial t^{*}}+q^{*} \frac{\partial T^{*}}{\partial x^{*}}=F^{\prime} N_{r} \sin \pi t^{*}-F^{\prime} N_{L} T^{*}
$$


where

$$
\begin{aligned}
T^{*} & \equiv \frac{T-T_{a}}{S_{o} / U_{o}}, \\
x^{*} & \equiv x / L_{c} \\
q^{*} & \equiv q /\left(U_{o} / \rho C_{p}\right) \\
t^{*} & \equiv t / \omega \\
N_{c} & \equiv \rho C_{p} \delta / U_{0} \omega \\
N_{r} & \equiv G_{m} / S_{o} \\
N_{L} & \equiv U_{L} / U_{o} .
\end{aligned}
$$

Here, $S_{o}$ is the solar constant, $1.353 \mathrm{kw} / \mathrm{m}^{2}, U_{o}$ is the reference heat loss coefficient and is usually taken as the value for collector with single glazing, and $L_{c}$ is the length of the absorber plate. It can be seen that normalization of eqn (1) results in four demensionless groups which characterize the thermal performance of the absorber: namely, $N_{c}, N_{r}, F^{\prime}$ and $N_{L}$. $N_{c}$ has the physical meaning of the rate of heat storage by the fluid inside the absorber plate and which controls the thermal transient behavior. $N_{r}$ represents the radiation intensity incident upon the collector. $N_{L}$ represents the quantity of heat loss from the absorber plate.

Similarily, applying energy balance to the storage tank for the condition of no water drawn-off we obtain:

$$
\frac{\partial \theta}{\partial t}+\frac{A_{c} H_{T}}{V_{T}} q \frac{\partial \theta}{\partial y}=\frac{k_{f}}{\rho C_{p}} \frac{\partial^{2} \theta}{\partial y^{2}},
$$

where $A_{c}$ is the absorber surface area, $V_{T}$ is the water volume in tank. $H_{T}$ is the water hieght in the tank. Equation (3) can be normalized to

$$
\frac{\partial \theta^{*}}{\partial t^{*}}+\frac{1}{N_{T}} q^{*} \frac{\partial \theta^{*}}{\partial y^{*}}=\frac{1}{P_{e}} \frac{\partial^{2} \theta^{*}}{\partial y^{* 2}},
$$

where

$$
\begin{aligned}
\theta^{*} & \equiv \frac{\theta-T_{a}}{S_{o} / U_{o}} \\
y^{*} & \equiv y / H_{T} \\
t^{*} & \equiv t / \omega \\
N_{T} & \equiv \frac{\rho C_{p} V_{T}}{A_{c} U_{o} \omega} \\
P_{e} & \equiv \frac{\rho C_{p} H_{T}^{2}}{k_{f} \omega} .
\end{aligned}
$$

It is seen that the normalization of eqn (3a) results in two dimensionless groups which govern the performance of the storage tank: $N_{T}$ and Pe. $N_{T}$ is a number physically representing the ratio of the water quantity in the tank to the absorber surface area and has long been shown very important in describing the collector performance. Thus $N_{T}$ can be called the "tank number". Pe is a Peclet number which illustrates the ratio of convective to conductive heat transfer along the fluid flow inside the storage tank. Mathematically, the last term of eqn (3a) can be ignored with negligible errors if the value of $\mathrm{Pe}$ is larger (larger than 50). In general, the values of $\mathrm{Pe}$ in most designs are much larger than 50 , therefore eqn (3a) can be simplified to

$$
\frac{\partial \theta^{*}}{\partial t^{*}}+\frac{1}{N_{T}} q^{*} \frac{\partial \theta^{*}}{\partial y^{*}}=0
$$

\section{Momentum balance}

The thermosyphon head induced in the collector can be evaluated by the integration of the density curve as shown in Fig. 1. The result can be written as

$$
\begin{gathered}
H_{t}=\int_{0}^{H_{1}}\left[S G\left(T_{1}\right)-S G(T)\right] \mathrm{d} z+H_{2}\left[S G\left(T_{1}\right)-S G\left(T_{2}\right)\right] \\
+\int_{H_{1}+H_{2}}^{H}\left[S G(\theta)-S G\left(T_{2}\right)\right] \mathrm{d} z
\end{gathered}
$$

where for water $S G(T)=-4.05 \times 10^{-6} T^{2}-3.906 \times$ $10^{-5} T+1.0002556, T$ in ${ }^{\circ} \mathrm{C}$. The above equation can be normalized to the form:

$$
\begin{aligned}
h_{t}^{*}=\int_{0}^{h \dagger}\left[S G\left(T_{1}^{*}\right)-S G\left(T^{*}\right)\right] \mathrm{d} z^{*} & +h_{2}^{*}\left[S G\left(T_{1}^{*}\right)-S G\left(T_{2}^{*}\right)\right] \\
& +\int_{h^{*}+h_{2}^{*}}^{1}\left[S G\left(\theta^{*}\right)-S G\left(T_{2}^{*}\right)\right] \mathrm{d} z^{*},
\end{aligned}
$$

where $h_{t}^{*} \equiv H_{t} / H, z^{*} \equiv z / H, h_{1}^{*} \equiv H_{1} / H, h_{2}^{*} \equiv H_{2} / H$.

The frictional loss due to fluid motion in the collector can be written as:

$$
H_{f}=f_{c} \frac{L_{c}}{D_{c}} \frac{v_{c}^{2}}{2 g}+f_{p} \frac{L_{p}}{D_{p}} \frac{v_{p}^{2}}{2 g}+E_{T} \frac{v_{p}^{2}}{2 g},
$$

where $D_{c}$ is the hydraulic diameter of the parallel-plate absorber and equal to $2 \delta W /(\delta+W), v_{p}$ is the velocity in the connecting pipe, $W$ is the width of the absorber. $f_{c}$ and $f_{p}$ are frictional coefficients in the absorber plate and in the connecting pipe respectively, $E_{T}$ is the loss factor due to valves, fittings and bends etc. Since the flow is assumed laminar in the collector, eqn (7) can be rewritten in the following normalized form after introducing the Fanning friction factor.

$$
h_{f}^{*}=3 \nu^{*} q^{*} / N_{f}+q^{* 2} /\left(2 N_{e}\right),
$$

where

$$
\begin{aligned}
& \begin{array}{l}
h_{f}^{*} \equiv H_{f} / H \\
\nu^{*} \equiv \nu / \nu_{o}
\end{array} \\
& 1 / N_{f} \equiv \frac{\nu_{o}}{g H}\left[\frac{L_{c}^{2}}{\delta^{2}}+\frac{8 \pi}{3}\left(\frac{\delta L_{p}}{A_{p}}\right)\left(\frac{A_{c}}{A_{p}}\right)\right] \frac{U_{o}}{\rho C_{p} \delta} \\
& 1 / N_{e} \equiv \frac{E_{T}}{g H}\left(\frac{A_{c}}{A_{p}}\right)^{2}\left(\frac{U_{o}}{\rho C_{p}}\right)^{2} .
\end{aligned}
$$

$\nu_{o}$ is the kinematic viscosity and usually taken at the ambient temperature. It is seen from eqn (8) that the 
magnitude of $N_{f}$ and $N_{e}$ represent the flow resistance of the collector. Since the thermosyphon head is balanced by the frictional head, equating eqns (6) and (8), we obtain the momentum equation:

$$
\begin{aligned}
& 3 \nu^{*} q^{*} / N_{f}+q^{* 2} / 2 N_{e}=\int_{0}^{h^{*}}\left[S G\left(T_{1}^{*}\right)-S G\left(T^{*}\right)\right] \mathrm{d} z^{*} \\
&++h_{2}^{*}\left[S G\left(T_{1}^{*}\right)-S G\left(T_{2}^{*}\right)\right]+ \\
&+\int_{h \uparrow+h_{2}^{*}}^{1}\left[S G\left(\theta^{*}\right)-S G\left(T_{2}^{*}\right] \mathrm{d} z^{*} .\right.
\end{aligned}
$$

In order to take into account the influence of temperature on the kinematic visocity, the viscosity $\nu^{*}$ in eqn (9) is taken at the mean temperature of the absorber, $\left(T_{1}^{*}+T_{2}^{*}\right) / 2$.

\section{Descriptions of collector performance}

The collector performance can be described in a number of ways. The solutions to the above equations could be used to calculate the specific instantaneous efficiency $\eta$ which is defined as

$$
\eta=\frac{m C_{p}\left(T_{2}-T_{1}\right)}{A_{c} S}=\frac{q^{*}\left(T_{2}^{*}-T_{1}^{*}\right)}{N_{r} \sin \pi t^{*}},
$$

where $m$ is the mass flowrate through the collector. It should be noted here that the specific collector effiiency is not the actual overall collector efficiency as defined in the presentation of the collector performance tests. Instead, the specific efficiency is defined based on the amount of solar incident radiation converted into the thermal energy at the black surface of the absorber. For convenience, we may define the specific mean efficiency as

$$
\bar{\eta}=\frac{\int_{0}^{\omega} m C_{p}\left(T_{2}-T_{1}\right) \mathrm{d} t}{\int_{0}^{\omega} A_{c} S \mathrm{~d} t}=\frac{\int_{0}^{1} q^{*}\left(T_{2}^{*}-T_{1}^{*}\right) \mathrm{d} t^{*}}{\int_{0}^{1} N_{r} \sin \pi t^{*} \mathrm{~d} t^{*}},
$$

and define the mean flowrate as

$$
\bar{q}^{*}=\int_{0}^{\omega} q^{*} \mathrm{~d} t / \omega=\int_{0}^{1} q^{*} \mathrm{~d} t^{*} .
$$

The final mean tank temperature is defined as

$$
\bar{\theta}_{f}^{*}=\frac{\bar{\theta}_{f}-T_{a}}{S_{o} / U_{o}} .
$$

From the above analysis, it is seen that the collector performance can be described by the three energy and momentum equations, eqns (2), (4) and (9). In addition, another relation concerning the collector geometry also should be satisfied, i.e.

$$
h_{1}^{*}+h_{2}^{*}+h_{T}^{*}=1 .
$$

Thus the present analysis comes out with ten dimensionless groups or system characteristic parameters which will uniquely determine the system performance of the collector: namely, $N_{c}, F^{\prime}, N_{T}, \mathrm{Pe}, N_{L}, N_{r}, N_{f}, N_{e}, h_{1}^{*}$ and $h_{2}^{*}$. In other words, the specific mean collector efficiency or flowrate is uniquely a function of these ten parameters. This development forms the basis of the similarity theory of the solar collector with natural circulation. Through these parameters, the collector performance can be clearly understood and a general design criteria can be drawn.

\section{NUMERICAL SOLUTION}

Equations (2), (4) and (9) can only be solved numerically with the initial condition that the water temperature is uniform and assumed to be the same as the ambient temperature for simplicity. Therefore, the final mean tank temperature can be evaluated by the summation of the temperature rise due to the total amount of energy flowing into the tank and the initial ambient temperature, i.e.

$$
\bar{\theta}_{f}=\frac{\int_{0}^{\omega} \rho C_{p} A_{c} q\left(T_{2}-T_{1}\right) \mathrm{d} t}{\rho C_{p} V_{T}}+T_{a},
$$

where $T_{a}$ is assumed constant and equal to $20^{\circ} \mathrm{C}$ in the present analysis. Substituting eqn (15) and combining with eqn (13) we obtain

$$
\bar{\theta}_{f}^{*}=(\pi / 2)\left(N_{r} / N_{T}\right) \bar{\eta} .
$$

Equation (16) shows that the final mean water temperature in the storage tank is linearly proportional to $N_{r} N_{T}$ and $\bar{\eta}$. The smaller the tank number, the higher the final mean temperature; and the higher the incident radiation the higher the final mean temperature.

Rewriting the governing equations, eqn (2), (4) and (9), into the finite difference form and applying the finite difference technique, the numerical solutions can be found when proper values of the ten parameters are given. Since the connecting pipes are assumed wellinsulated, at any instant the inlet temperature of the storage tank will always equal to the exit temperature of the absorber plate, and the exit temperature of the storage tank will always equal to the inlet temperature of the absorber. Since the transient behavior of the heat transfer inside the connecting pipe is ignored, the temperatures at the exits of the absorber and the storage tank will immediately transmit to the inlets of the tank and the absorber respectively. This is the boundary conditions to be obeyed in numerical calculation. The numerical computations are carried out by dividing each of the absorber and the tank into sixteen segments and using the time step of 0.008 . From the results obtained, it is shown that twelve segments are satisfactory for the present computation. A CDC 6000 Cyber 72 digital computer was used in the computational work. The calculation starts with the initial condition: $\theta^{*}=0$ and $T^{*}=0$ at $t^{*}=0$, and stops at $t^{*}=1$ or when $q^{*}=0$. 


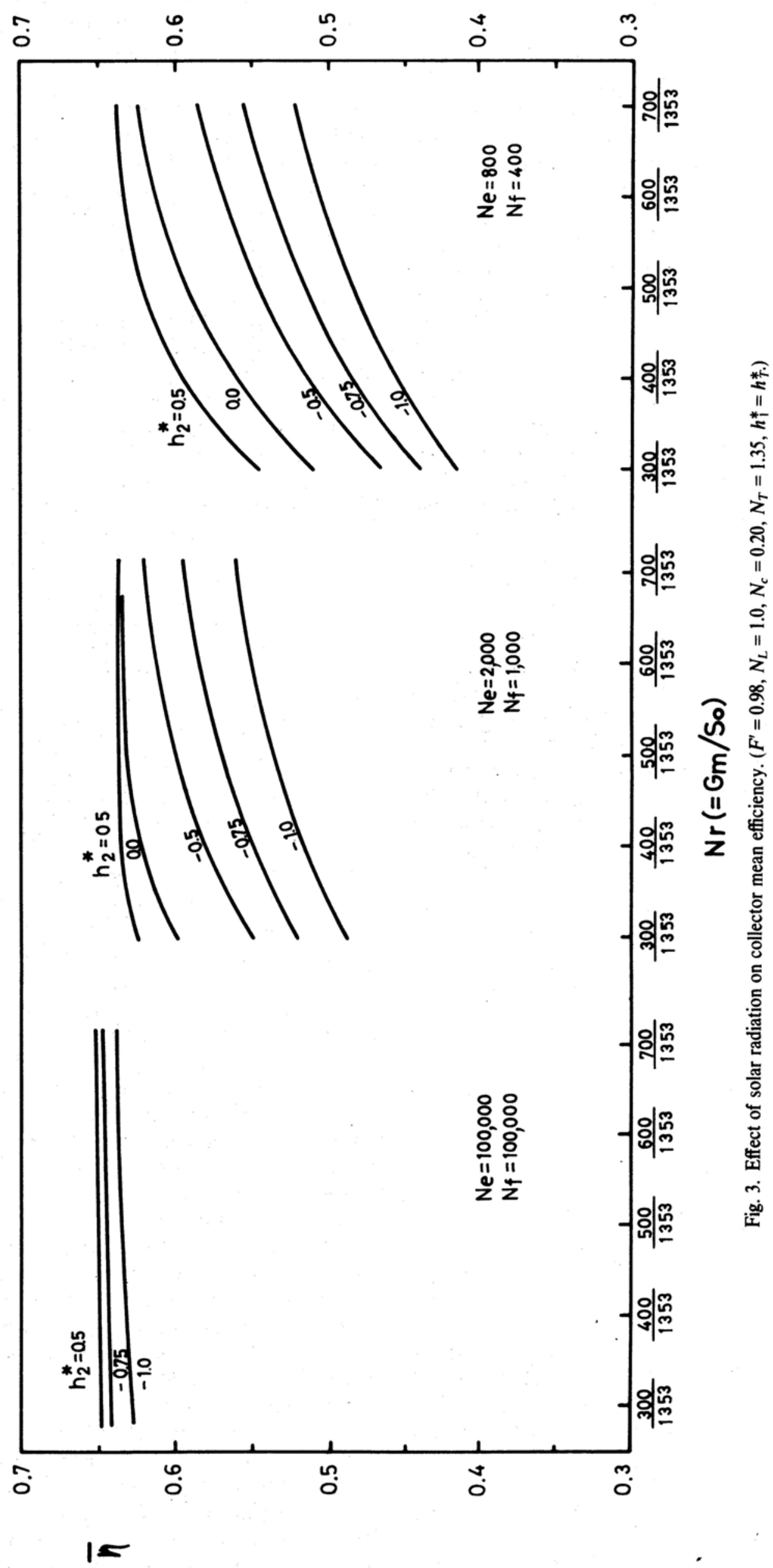




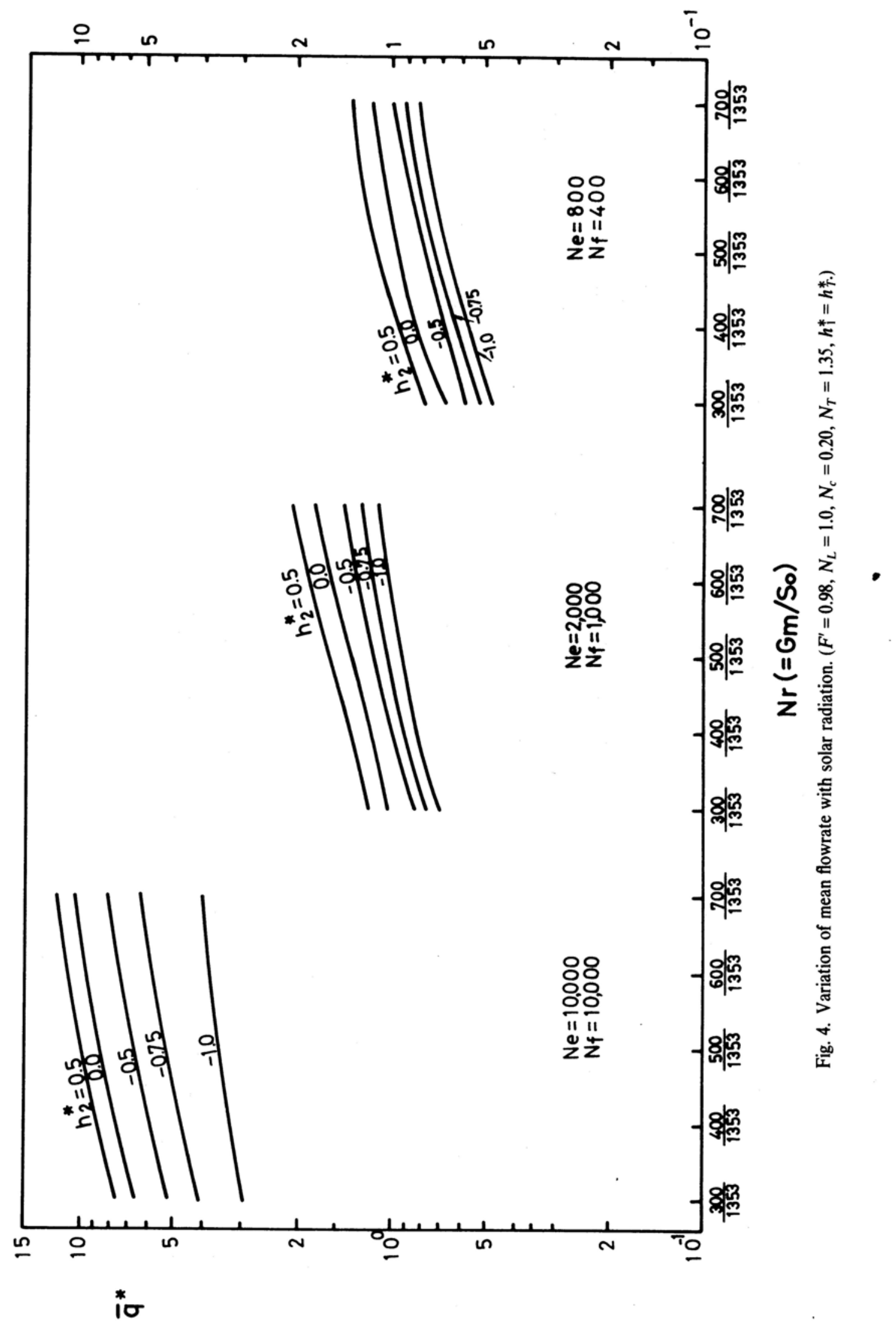




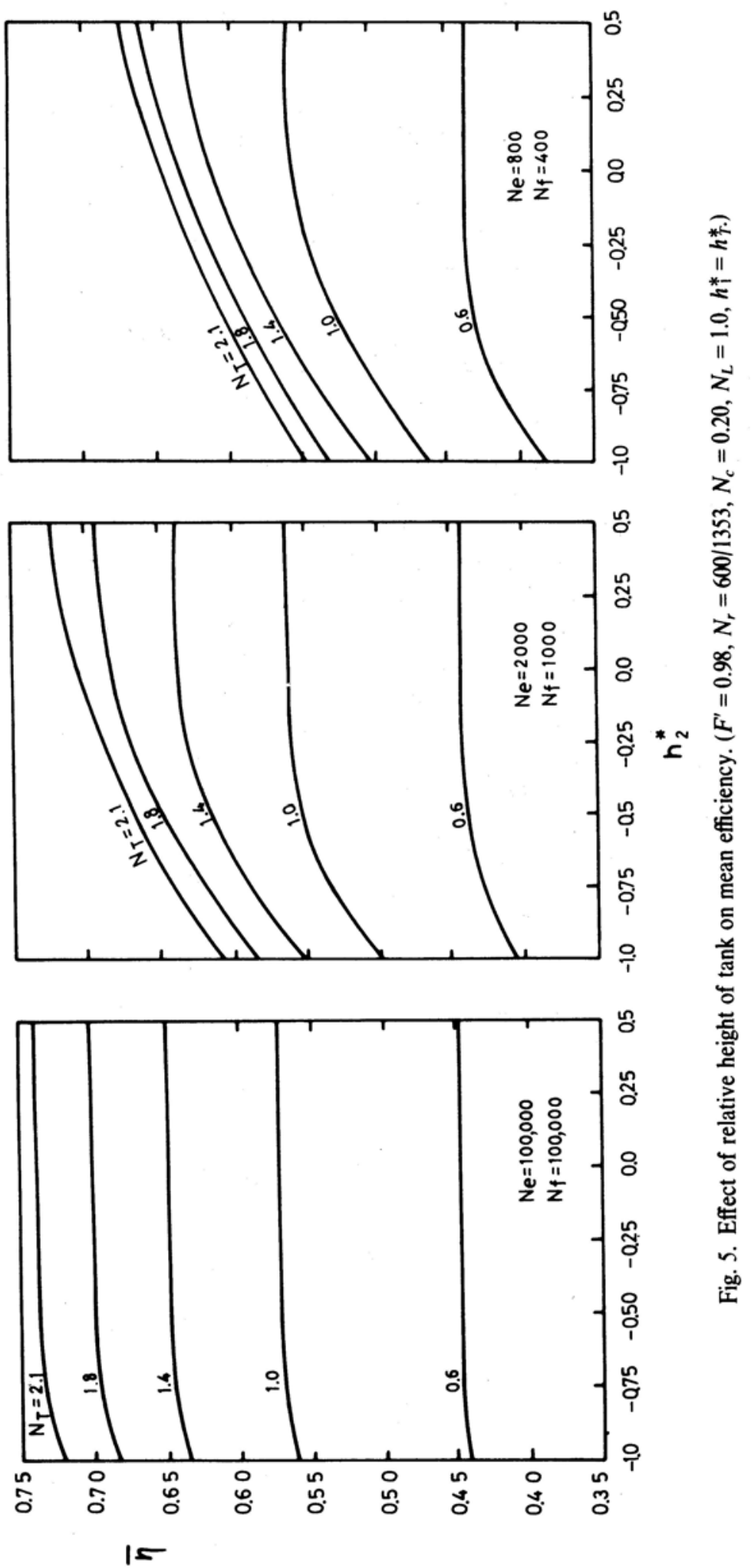


RESULTS AND DISCUSSIONS

For simplicity, the present analysis assumes that $h_{1}^{*}=$ $h_{T}^{*}$ and $\mathrm{Pe}$ can be ignored since the values of $\mathrm{Pe}$ are usually larger than 50 in most designs (it has been numerically shown in the present computation that the results do not make any difference when $\mathrm{Pe}$ is larger than 50). Therefore there are only eight system characteristic parameters to be considered.

Figure 3 shows that the mean efficiency increases with increasing incident solar radiation and increasing relative height of the tank. This is consistent with Ong's experiments [5]. However, it is very interesting to note that, as the values of $N_{e}$ and $N_{f}$ become very high (greater than $10^{5}$ ), i.e. the resistance to the fluid motion is very small, the efficiency tends to reach an equilibrium value and be independent of both the incident solar radiation and the relative height of the tank. Even at the case of $h_{2}^{*}=-1.0$, which means that the storage tank is sitting on the ground, the mean efficiencies are still independent of incident solar radiation and very close to each other (within 5 per cent deviation) for different relative heights of the tank. Therefore we can conclude that in order to reduce the influence of solar radiation due to the variations of seasons or weather conditions and obtain the highest efficiency, it is preferable to design a thermosyphon collector with $N_{e}$ and $N_{f}$ larger than $10^{5}$ and under this circumstance we may also let the storage tank sit on the ground to reduce the construction cost without sacrificing efficiency. This saturation phenomenon on efficiency is mainly attributed to the balance between flowrate and heat loss. It is seen that higher incident radiation and higher relative height of the storage tank causes to high thermosyphon head as well as flowrate, as shown in Fig. 4; higher flowrate then induces higher moving speed of the temperature wave front in the tank and consequently raises the inlet temperature of the absorber and relatively lowers the increase of efficiency. An equilibrium is finally reached and the efficiency approaches constant as shown in Fig. 3.

It is important to see how the relative height of the tank influences the collector performance. Figure 5 has clearly shown that the relative height of the storage tank has very little effect on the mean efficiency for collectors with $N_{e}$ and $N_{f}$ larger than $10^{5}$. Also, the influence on the mean efficiency is relatively small for smaller tank number. These results indicate that the relative height of the storage tank is mainly determined by the flow resistance of the collector. For ordinary size of parallel-plate absorber such as $3 \mathrm{ft} \times 6 \mathrm{ft} \times 1 / 10$ in. in which the frictional loss is very low, $N_{e}$ and $N_{f}$ are usually larger than $10^{5}$, therefore we may lower the height of the storage tank without sacrificing efficiency.

Since the tank number reflects the amount of water to be heated and stored in the tank with respect to the absorber surface area, it controls the water temperature and the efficiency. High tank number would result in lower water temperature as well as heat loss and therefore increases the efficiency as shown in Fig. 6. The rate of increase of efficiency with respect to the tank number is higher for higher values of $N_{e}$ and $N_{f}$. From Fig. 7 it also can be seen that the lower heat loss of the collector will increase the efficiency.

It is known that the amount of water contained in the absorber has some effect on the performance as shown in Fig. 8. The efficiency is found to decrease with increasing $N_{c}$ because higher $N_{c}$ means that more nonapplicable hot water is kept in the absorber.

As discussed previously, the flow resistance of the collector affects the flowrate as well as the efficiency.

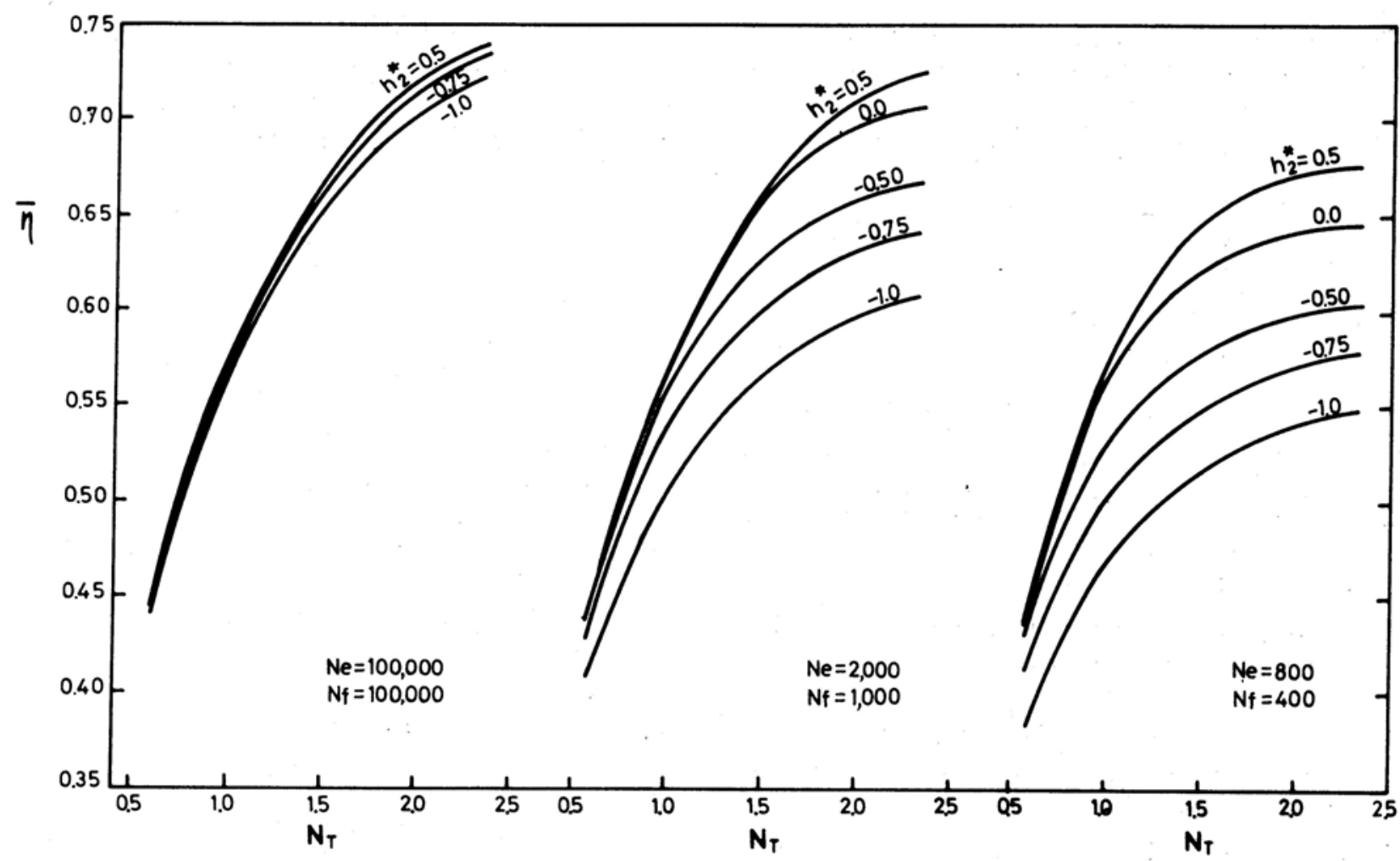

Fig. 6. Effect of tank size on mean efficiency. $\left(F^{\prime}=0.98, N_{r}=600 / 1353, N_{c}=0.20, N_{L}=1.0, h_{1}^{*}=h_{7}^{*}\right.$. 


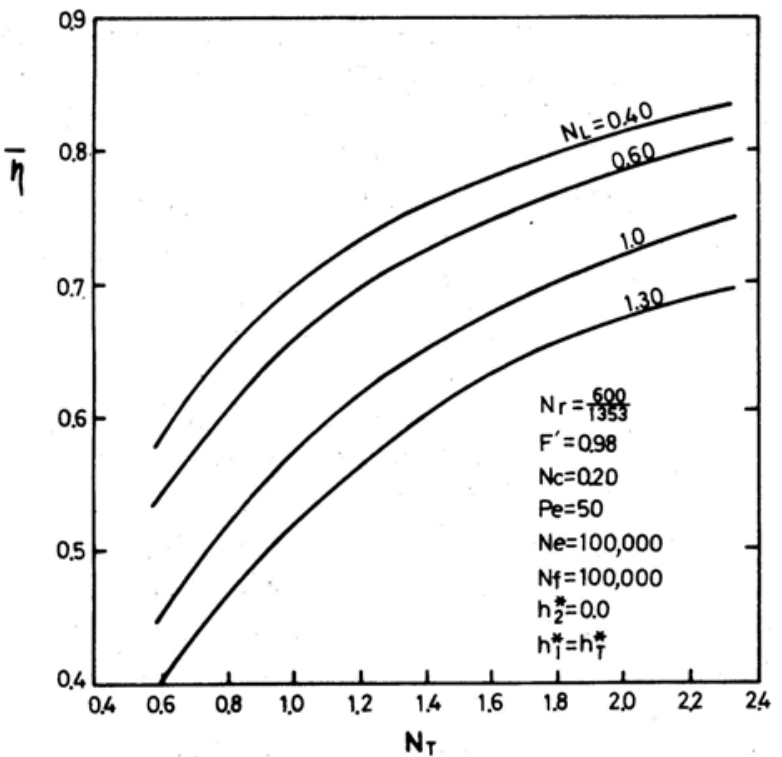

Fig. 7. Variation of mean efficiency collector heat loss.

When both $N_{e}$ and $N_{f}$ are larger than $10^{5}$, the resulting high flowrate will cause to a relative increase of the inlet temperature at the absorber due to fast moving of the temperature front in the tank. An equailibrium condition will be eventually reached as shown clearly in Fig. 9, and the efficiency will no longer increase with $N_{e}$ and $N_{f}$. Figure 10 describes the instantaneous motion of the temperature wave front in the tank. For the case of low flow resistance (high $N_{e}$ and $N_{f}$ ), the temperature wave front moves very fast. But at the end of the performance near sunset hours, the temperature distribution in the tank tends to form a peak near the top of the tank, especially for the case of low flowrate. This temperature peak would destroy the condition of thermal stratification in the tank as assumed previously and mixing process would take place at the top of the tank. Under this condition, the thermosyphon head would change and the performance prediction would result in some errors. However, as this phenomenon usually occurs at the end of the performance, the resulting error would not be too serious. The occurence of the mixing phenomenon also can

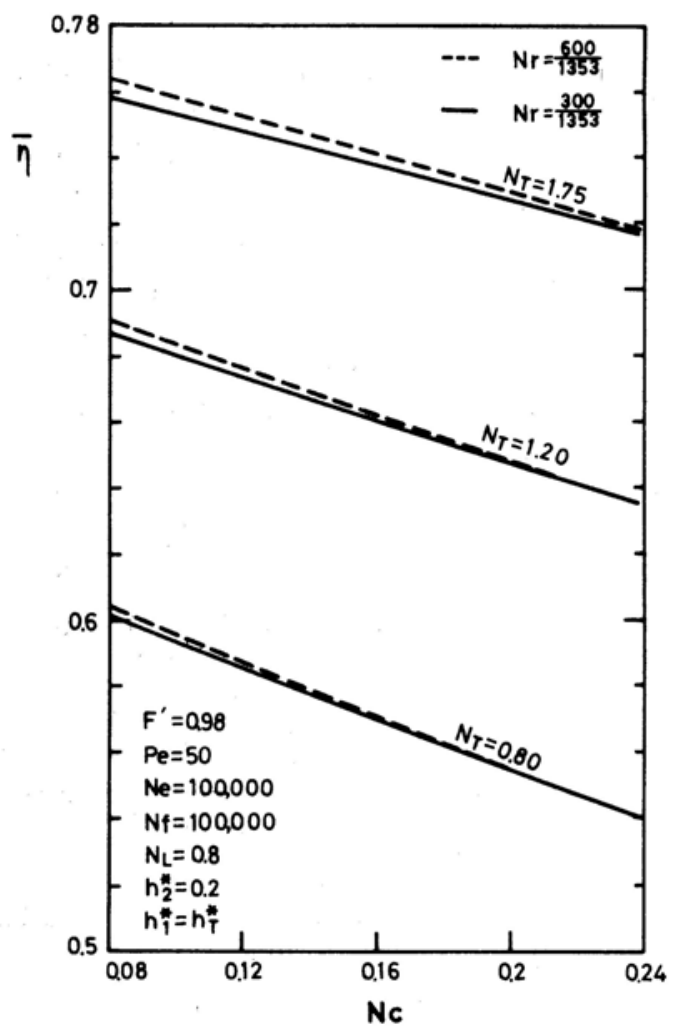

Fig. 8. Variation of mean efficiency with absorber size $\left(N_{c}\right)$.

be seen from Fig. 11. In the present analysis the mixing effect is ignored, so the computation is terminated at the time when the negative thermosyphon head resulting from the peak temperature in the tank is equal to the positive thermyphon head as shown in Fig. 11, i.e. when $q^{*}=0$. More work should be carreid out to investigate the effect of mixing.

\section{CONCLUSIONS}

The similarity theory of solar water heater with natural circulation is developed in the present paper. Ten dimensionless groups or system characteristic parameters which uniquely determine the system performance are derived. The numerical solutions to the governing equations has shown that the mean efficiency

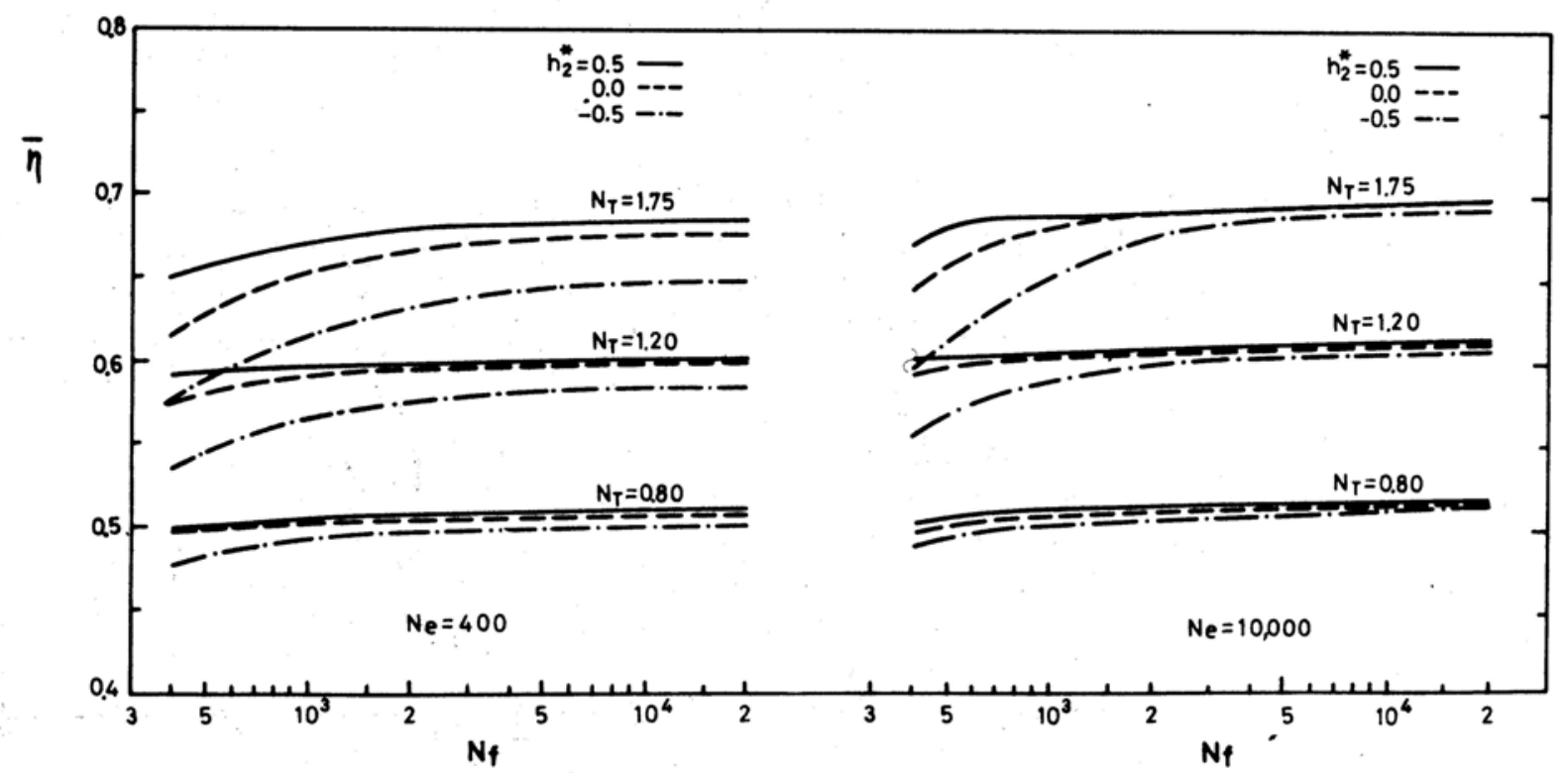

Fig. 9. Effect of flow resistance on mean efficiency. $\left(F^{\prime}=0.98, N_{c}=0.20, N_{r}=600 / 1363, N_{L}=1.0\right.$.) 

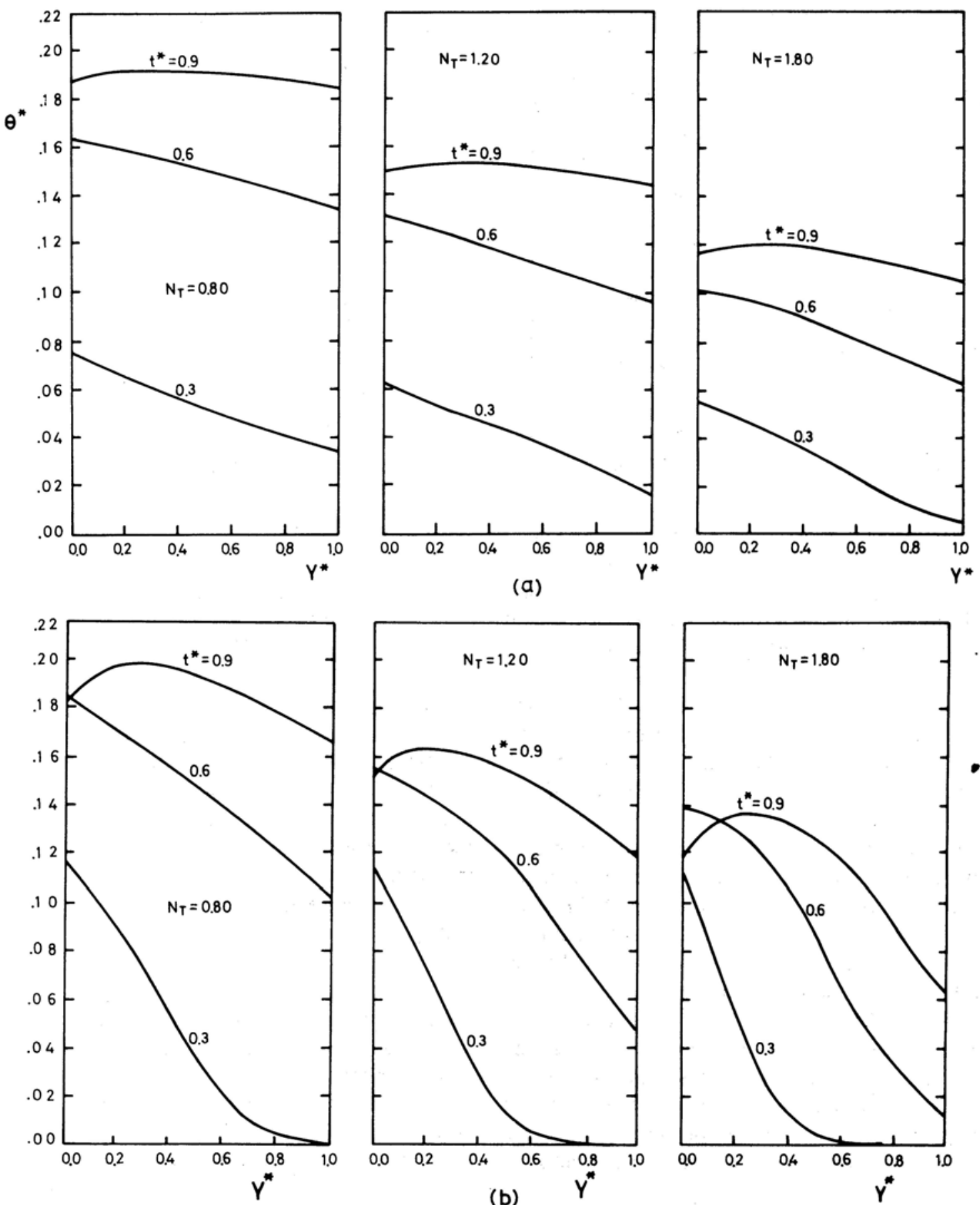

Fig. 10. Moticn of temperature wave front in storage tank. $\left(F^{\prime}=0.98, N_{c}=0.20, N_{L}=1.0, N_{r}=600 / 1353\right.$, $h_{2}^{*}=0.2$.) (a) $N_{e}=N_{f}=20,000$, (b) $N_{e}=N_{f}=1500$.

increases with increasing incident solar radiation and relative height of the tank. However, for high $N_{e}$ and $N_{f}$ (larger than $10^{5}$ which are of the values for ordinary parallel-plate absorber), the efficiency appears to be independent of the incident radiation and the relative height of the tank. This points out a very important design criteria which differs from other investigators' in that, the tank may be designed to sit on the floor without sacrificing the efficiency for parallel-plate absorber since its $N_{e}$ and $N_{f}$ are usually larger than $10^{5}$. This also indicates that the resistance to flow is the most important factor which should be carefully considered in the design of a solar collector with thermosyphon cycle. Low flow resistance would largely favor the collector performance in both the mean efficiency and the structural design of the relative height of the tank. The analysis also shows that higher tank number and lower $N_{c}$ and $N_{L}$ increase the mean efficiency. From the motion of the temperature wave front in the tank, we also note that the mixing process near the top of the tank will take place and affect the performance as the flowrate is low. Since the present analysis ignores the effects of water heating load (i.e. hot water drawn-off) during daytime, mixing in the tank, heat loss from the tank and piping and transient behavior in the pipes, etc. some analytical errors are inevitable and further theoretical and experimental investigations are required.

Acknowledgement-The author is grateful to Mr. H. T. Hsu for his assistance in the numerical calculation. He also thanks Aiteh Engineering Co. for providing financial support. 


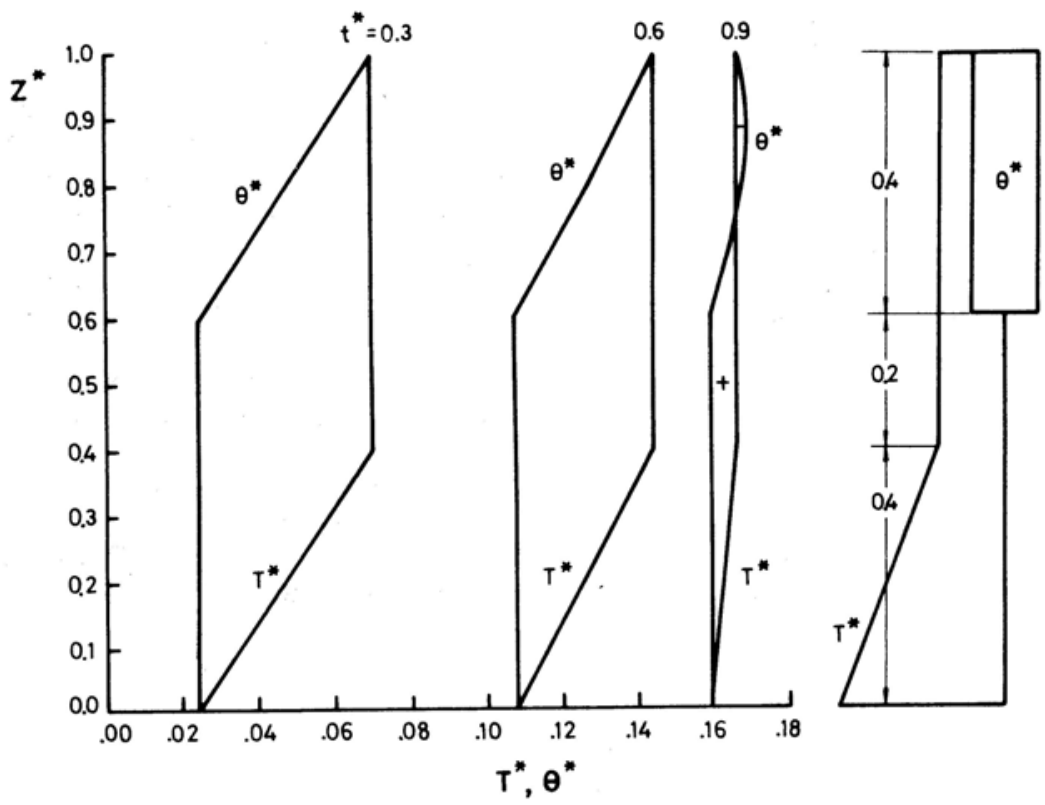

(a)
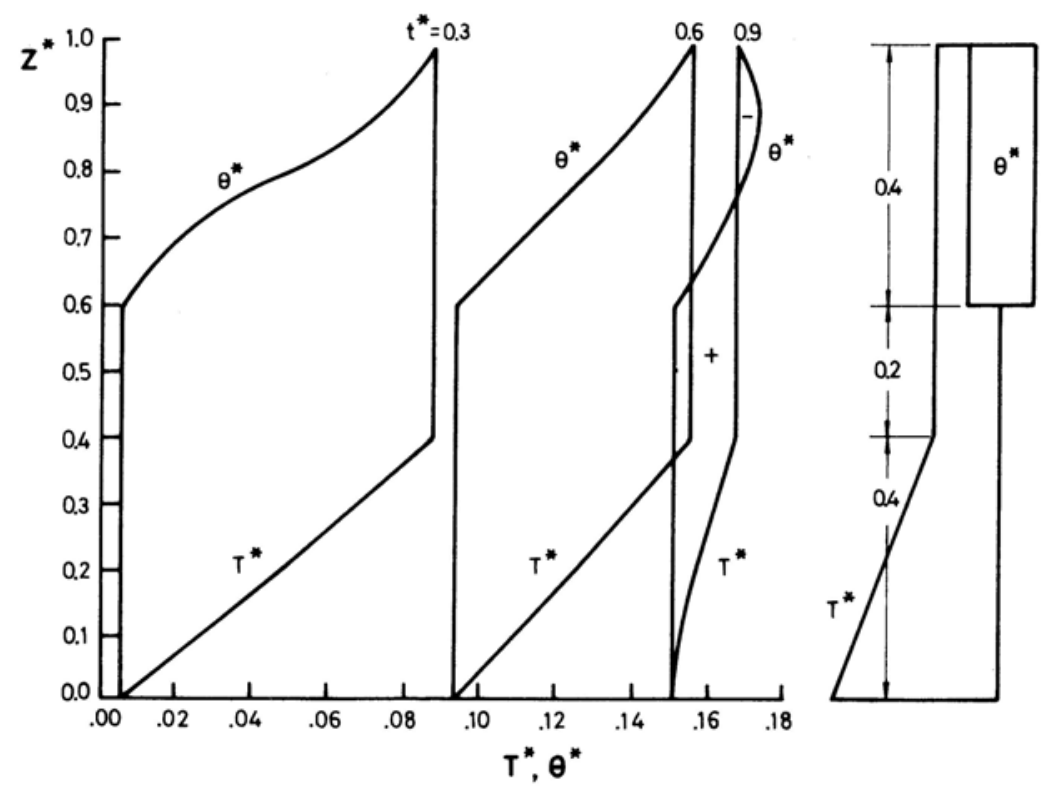

(b)

Fig. 11. Instantaneous temperature distributions of collector system. $\left(F^{\prime}=0.98, N_{c}=0.20, N_{L}=0.6, N_{r}=600 / 1353\right.$,

$$
N_{T}=1.20 \text {.) (a) } N_{e}=N_{f}=20,000 \text {, (b) } N_{e}=N_{f}=5000 \text {. }
$$

\section{NOMENCLATURE}

$A_{c}$ absorber surface area, $\mathrm{m}^{2}$

$A_{p}$ cross-sectional area of connecting pipe, $\mathrm{m}^{2}$

$B_{i}$ Biot number $\left(=h_{i} e / k_{c}\right)$

$C_{p}$ heat capacity of fluid, cal $/ \mathrm{g}-\mathrm{C}$

$\underline{\theta}$ temperature distribution in tank, C

$\bar{\theta}_{f}$ final mean tank temperature, $\mathrm{C}$

$\hat{\theta}_{f}^{*}$ dimensionless final mean temperature

$D_{p}$ diameter of connecting pipe, $\mathrm{m}^{2}$

$\delta$ spacing of parallel-plate absorber, $\mathrm{m}$

$E_{T}$ loss factor due to valves, fittings, and bends etc.

$\eta$ instantaneous efficiency

$\bar{\eta}$ mean efficiency

$e$ thickness of absorber plate material, $\mathrm{m}$

$F^{\prime}$ absorber geometry efficiency factor

$f_{c}$ Fanning frictional factor in connecting pipe

$f_{p}$ Fanning frictional factor in absorber

$G_{m}$ converted solar energy at solar noon, $\mathrm{kW} / \mathrm{m}^{2}$

$H_{T}$ height of water in tank, m

$H_{t}$ thermosyphon head, $\mathrm{m}$

$H_{1}$ absorber height, $\mathrm{m}$

$\mathrm{H}_{2}$ relative height of tank above absorber, m
$h_{T}^{*}$ dimensionless water height in tank

$h_{i}$ heat convection coefficient inside the absorber, $\mathrm{kW} / \mathrm{m}^{2}$ C

$h_{f}^{*}$ dimensionless frictional head

$h_{t}^{*}$ dimensionless thermosyphon head

$h_{1}^{*}$ dimensionless height of absorber

$h_{2}^{*}$ dimensionless relative height of tank above absorber

$k_{c}$ thermal conductivity of absorber plate material, $\mathrm{kW} / \mathrm{m}^{2} \mathrm{C} / \mathrm{m}$

$k_{f}$ thermal conductivity of fluid, $\mathrm{kW} / \mathrm{m}^{2} \mathrm{C} / \mathrm{m}$

$L_{c}$ absorber length, $\mathrm{m}$

$L_{p} \quad$ connecting pipe length, $\mathrm{m}$

$m$ total mass flowrate, $\mathrm{kg} / \mathrm{hr}$

$N_{c}=\rho C_{p} \delta / U_{o} \omega$ (absorber number)

$N_{e}$ loss number

$N_{f}$ friction number

$N_{L}$ heat loss number

$N_{r}$ insolation number $\left(=G_{m} / S_{o}\right)$

$N_{T}$ tank number $\left(=\rho C_{p} V_{T} \mid A_{c} U_{o} \omega\right)$

$\nu_{o}$ reference kinematic viscosity, $\mathrm{m}^{2} / \mathrm{hr}$

$\nu^{*}$ dimensionless kinematic viscosity

$\omega$ sunshine duration, $\mathrm{hr}$ 
Pe Peclet number in tank $\left(=\rho C_{p} H_{T}^{2} / \mathrm{k}^{\omega}\right)$

$q$ volumetric flowrate per unit area of absorber, $\mathrm{kg} / \mathrm{hr}$ $m^{2}$

$q^{*}$ dimensionless volumetric flowrate

$S$ solar energy actually converted into heat energy at the absorber surface, $\mathrm{kW} / \mathrm{m}^{2}$

$S G$ specific gravity of fluid

$S_{o}$ solar constant, $1.353 \mathrm{kw} / \mathrm{m}^{2}$

$T$ local fluid bulk temperature in absorber, $\mathrm{C}$

$T_{a}$ ambient temperature, $\mathrm{C}$

$t$ time, hr

$U_{L}$ heat loss coefficient, $\mathrm{kw} / \mathrm{m}^{2} \mathrm{C}$

$U_{o}$ reference heat loss coefficient, $\mathrm{kw} / \mathrm{m}^{2} \mathrm{C}$

$V_{T}$ total water volume in tank, $\mathrm{m}^{3}$

$v_{c}$ fluid velocity in absorber, $\mathrm{m} / \mathrm{hr}$

$x, y, z$ coordinates, $\mathrm{m}$

$x^{*}, y,{ }^{*} z^{*}$ dimensionless coordinates
2. D. J. Close, The performance of solar water heaters with natural circulation. Solar Energy 6, 33 (1962).

3. C. L. Gupta and H. P. Garg, System design in solar water heater with natural circulation. Solar Energy 12, 163 (1968).

4. K. S. Ong, A finite-difference method to evaluate the thermal performance of a solar water heater. Solar Energy 16, 137 (1974).

5. K. S. Ong, An improved computer program for the thermal performance of a solar water heater. Solar Energy 18, 183 (1976).

6. G. Grossman, A. Shitzer and Y. Zvirin, Heat transfer analysis of a flat-plate solar energy collector. Solar Energy 19, 493 (1977).

7. A. Cabelli, Storage tanks-a numerical experiment. Solar Energy 19, 45 (1977).

8. Z. Lavan and J. Thompson, Experimental study of thermally stratified hot water storage tanks. Solar Energy 19, 519 (1977).

1. J. C. McVeigh, Sun Power, Chap. 9. Pergamon Press, Oxford (1977). 